\title{
FULL-DAY SIMULATION OF FIELD ENGINEERING OPERATIONS
}

\author{
Nicola Wong, Mathias Kern, Anne Liret, Raphael Dorne
}

Applied Research, BT

Adastral Park, Martlesham, Ipswich IP53RE

nicola.wong@bt.com, mathias.kern@bt.com, anne.liret@bt.com,raphael.dorne@bt.com

\begin{abstract}
This paper describes an on-going project on a full-day simulation of field engineering operations, enabling analysis of a company's capability to cope with changes in work demand and labour supply. This case study incorporates the operational dynamics in the scheduling process, illustrating a schedule of work for service delivery in BT Group plc (BT) over the course of an operational day. These jobs range from repairing existing network cables; installing new infrastructure; connecting cities to the internet and beyond. In this project, we model our operations using a discrete event simulation approach, adding a number of key in-day disturbances which are considered turbulent to BT's service delivery. Furthermore, we discuss the current development state and future directions for this project.
\end{abstract}

Keywords: Discrete-Event Simulation, Resource Management, Scheduling, Operational Dynamics

\section{INTRODUCTION}

In many service organisations such as communication or utility companies, teams of field engineers deliver services to customers. For these field teams, it's a daily reality that things do not go as planned, e.g. work could take longer than expected or might not be completed due to certain constraints. Therefore, schedule planners need to frequently review and update work schedules for their engineering resources in order to continuously balance work demand with supply of labour. This enables teams to meet the often challenging delivery targets towards the end of the day.

When creating a simulation for service operations, it is important to model in-day dynamics to ensure a close alignment between simulated results and real life outcomes. This not only has the potential to facilitate the verification and improvement of existing scheduling setups, but also allows the assessment of any impact to the operations when introducing new products or service offerings.

In this paper, we present a model that simulates both the start-of-day and in-day dynamics for a team of field engineers in the context of service delivery by BT.

\section{DYNAMIC NATURE OF FIELD OPERATIONS}

Service companies often utilise highly sophisticated scheduling systems for the allocation of work to their field resources. In BT, FieldSchedule (Liret et al, 2007 ; Owusu et al, 2017) is used to organise the large engineering teams in Technology's and Enterprise's field operations. A typical scheduler setup, also known as "predictive-reactive scheduling"(Chin S C, Appa I S and Robert G, 2003) includes a start-of-day scheduling engine run which produces an initial schedule for a full working day based on the work and resource information available very early in the morning. The system then updates this schedule with periodic "in-day" scheduling runs applying any latest changes in the field teams. This information can include the time taken to progress or complete a task, updates on travel duration; unplanned work being added and planned jobs being removed. Moreover, a day's plan can often go pear-shaped when job priorities are modified or availability of labour changes in the course of an operational day. In short, field operations can be very volatile, and a good scheduling system needs to manage these dynamics. 
In order to address to different service domains and their requirements, scheduling systems can be customised to a comprehensive set of configurations, by using what can be hundreds of parameters.

The setup and optimisation of scheduling configurations for specific scenarios is a non-trivial challenge that can have a profound impact on the service and productivity delivered. As live trials are often timeintensive and expensive, simulation plays an important role in evaluating different scheduling configurations. While traditionally, the focus has been on improving an individual schedule engine run at start-of-day, the aforementioned dynamic nature of field operations will require a simulation for an entire operational day - predictive schedules at start-of-day and actual outcomes at end-of-day are often very different. In the next section, we outline our approach to a full-day simulation of field operations.

\section{SIMULATION APPROACH}

We have developed a simulation system that not only simulates individual start-of-day scheduling scenarios but that also incorporates the key dynamics of operations with in-day scheduling. The endof-day results produced by this full-day simulation provide more realistic business insight than a startof-day simulation alone.

We have chosen a discrete event simulation approach (Liret A, 2009) in a combination with an Object Oriented (OO) program to model the change of states in a team of field resources and tasks on an operational day. The system's state is made up of a list of task instances, a list of resource instances and a 'current schedule' instance, maintained at all times. The state changes over a notion of simulated time according to the execution of a dynamically ordered list of events. An initial event list is created with events of a fixed time, such as the engineer signing on, the start-of-day schedule run event and the periodic in-day scheduling run events. Other events, such as engineer travel events, task begin/complete events and resource absences events, are dynamically added to the list. The event list always holds the events in a chronological order, and the simulation steps through the events in the same manner.

Figure 1 illustrates a sequence of key events which update the system states. Dark solid arrows represent the creation of an event. An event is executed when the simulation timeline arrives at the event start time. The execution of an event can create further events. Key information including start time of an event is often determined by its 'parent' event. Estimation functions may be applied to model these features of a generated event. For example, a 'task complete' event is generated following a 'task begins' event, and the time difference, i.e. the simulated task duration, is stochastically determined. The event list is maintained chronologically and kept up-to-date when an event inserts one or more offsprings at particular time points after its execution.

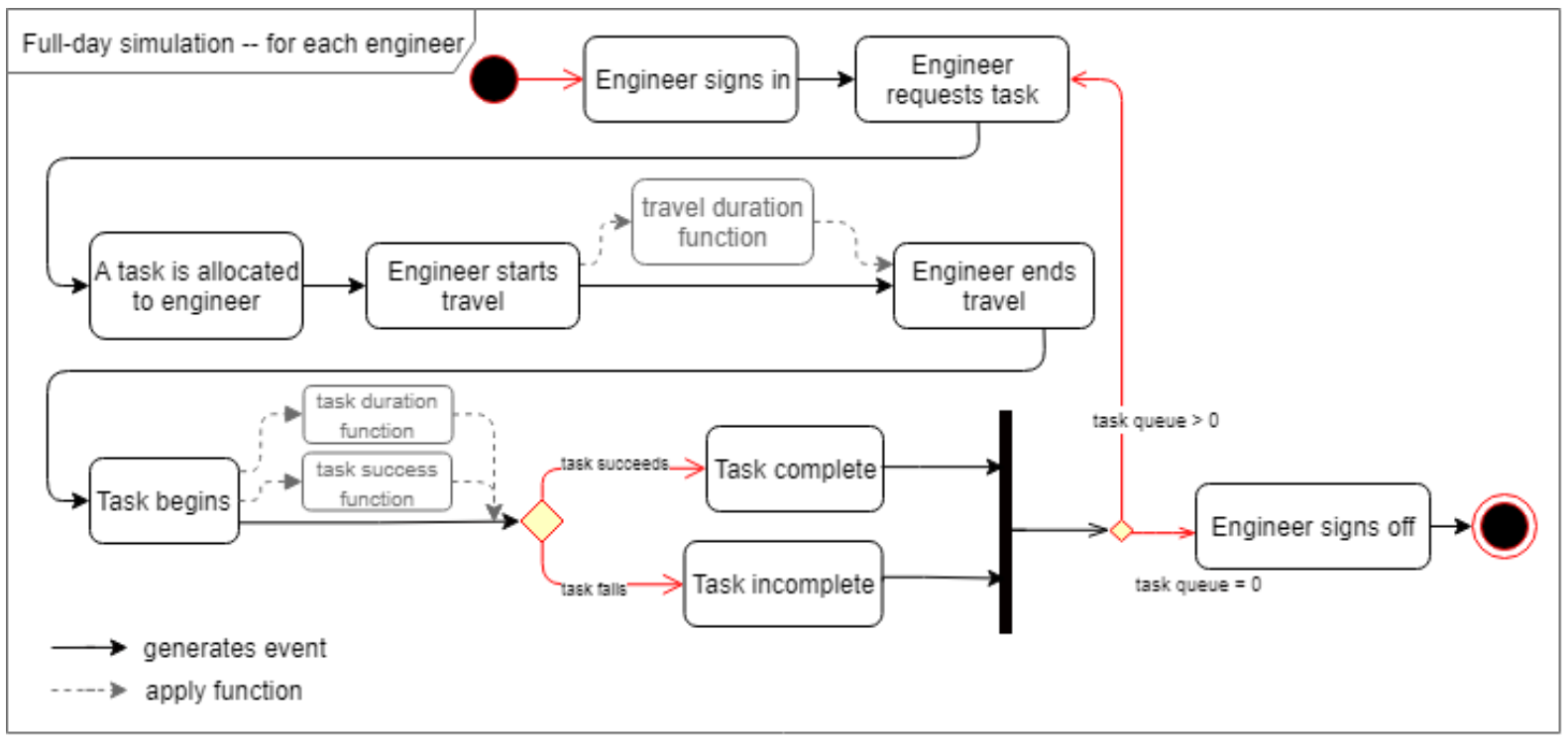

Figure 1 State diagram of event sequence upon each engineer's sign-on 
As outlined earlier, numerous events can impact the delivery of service by field engineering. Based on feedback from BT's operational teams, our main stakeholder in this use case, these turbulences are categorised in the following 6 types of unexpected events in our model:
i. Variation in actual task times
ii. Variation in actual travel times
iii. Success rates for the completion of work
iv. Unplanned absences of resources
v. Arrival of additional work
vi. Cancellations of existing work

One of the biggest challenges to model these stochastic disturbances is to determine the estimation function for their occurrences. As a case in point, The solid line on the graph shown in Figure 2 illustrates the distribution of historically observed task times for two typical types of work. We can see that the actual task time varies a lot. When a Gamma distribution is fitted to approximate the distribution of varying task times, i.e. dotted lines in Figure 2, we can see our estimation produces a close match to our historical data. So while a traditional single start-of-day schedule organises jobs bases on the an average of task times, the full-day simulation can produce a more realistic model by incorporating the varying task times as updates when frequently rescheduling the tasks.

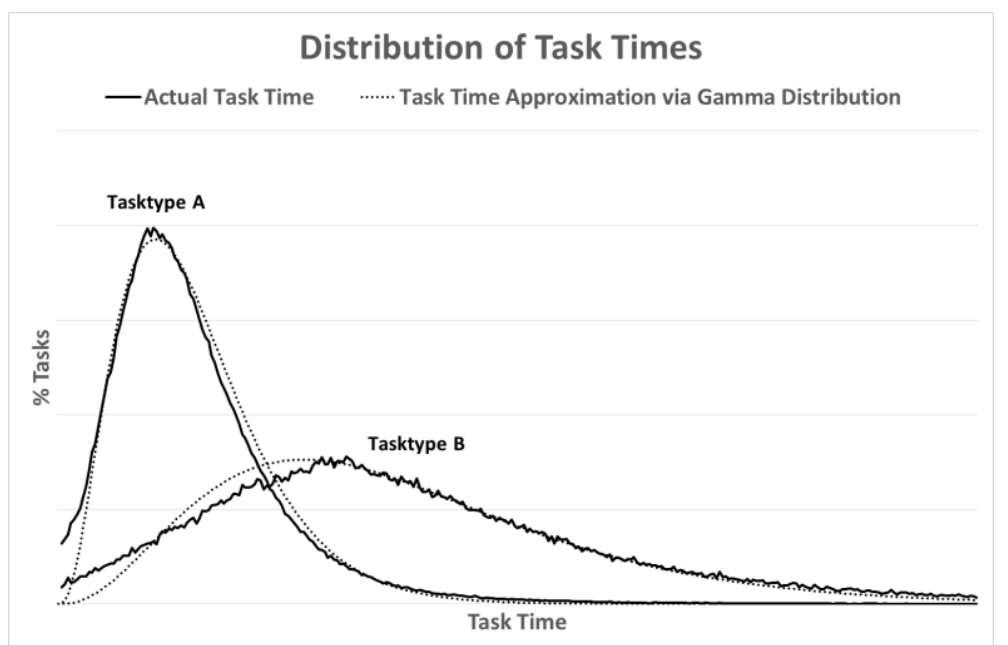

Figure 2 Distribution of actual task times and approximation using Gamma distributions

Likewise for the other the key disturbances listed above. A probability function is applied to their occurrences. These probabilism are determined by our analysis similar to this example with task times, based on the data contributed by our operational teams.

In this project, a simulated operational day starts at 00:00:00 and ends at 23:59:59 of the day. A start-of-day schedule is produced at 00:00:00. From that point on, a configurable number of periodic in-day schedules are generated throughout the simulated day, e.g. every 30 or 60 simulated minutes from 06:00:00 onwards. This means at start-of-day, the event list contains the different schedule run events, engineer sign-on events, new task arrival events that are generated stochastically, and more. The simulation retrieves the next event from the list, executes this event which induces an update in the state of resources, tasks and schedules, and inserts any new 'child' events to the event list. The simulation then moves on to the next chronological event. The simulation terminates once all events from the event list are executed for the simulated day. The simulation collects key statistics throughout its execution, building metrics such as average travel times, task completion rates, on-time service, and more.

This collection of data forms a central output of the simulation to assess the task coverage in a particular scheduling or service setup. In the occasion of testing a new work prioritisation business rule, the start-of-day schedule might not reflect the impact of such service setup. However, cases of increased travel or lower task completion could be modelled by a full-day simulation. Frankly, a better insight is presented. 


\section{CURRENT STATE AND FUTURE WORK}

Our work so far has focused on developing a system that is capable of simulating an entire operational day of a field engineering team. We have achieved this aim and are able to simulate both start-of-day and in-day events including key dynamics and disturbances such as variation in task times and task completion rates. We have been able to run initial tests which underline that outcomes envisaged by start-of-day schedules and outcomes delivered at the end-of-day can differ significantly.

The current technical implementation in Java is built in a modular infrastructure where the application is divided into four components: a data retrieval process, an API that queries the scheduling algorithm, a type-agnostic main simulation algorithm and a user-interface for displaying results. By having a dedicated simulation process that communicates with the scheduling engine via a generic API, we can plug different scheduling engines into the simulation and compare them. At the moment, we have employed FieldSchedule, an engine developed and maintained by our team, into the full-day simulation.

Going forward, our research and development work will focus on the following key areas:

1. Ability calibrate the simulation system, i.e. ensure that end-of-day results produced by our full-day simulation match observations from real life operations closely.

2. Provide meaningful suggestions to the business stakeholders on adjusting our work-force in BT utilizing simulation results

3. Enhance what-if and how-to simulation capabilities of the system

4. Enhance user interface to make the tool available to operational units

5. Build connections to plug-in other / third-party schedulers

\section{REFERENCES}

Chin S C, Appa I S and Robert G (2003) Dynamic scheduling I: simulation-based scheduling for dynamic discrete manufacturing. Conference Paper in Proceedings - Winter Simulation conference January 2004 DOI:10,1109/WSC.2003.1261590

Liret, A., Lesaint, D., Dorne, R., Voudouris, C. (2007). iSchedule, an optimisation toolkit for complex scheduling problems. Multidisciplinary International Scheduling Conference:Theory and Applications (MISTA), Paris, France, August 2007.

Owusu G. , Shakya S., Liret A., Mccormick A. (2017) Leveraging OR Techniques for Smarter Field Operations. Impact journal, vol 3(1), pp.18-21, March 2017. DOI: 10.1080/2058802X.2017.11964018

Liret, A., Workforce Dynamics Simulator in Service Operations Scheduling Systems, International Conference on Enterprise Information Systems CENTERIS 2009, Ofir, Portugal, October 2009

\section{AUTHOR BIOGRAPHIES}

NICOLA WONG is currently a research specialist in the Operational Transformation research theme at BT Applied Research. She received a BSc (Hons) Computer Science in 2016.

MATHIAS KERN is a senior research manager in BT's Applied Research team, and his research interests focus on the application of AI, optimisation and simulation techniques to real-life resource management problems. He gained his $\mathrm{PhD}$ in Computer Science from the University of Essex researching populationbased optimisation methods, and has been with BT since 2004 .

ANNE LIRET is currently research manager at BT's Applied Research. Her research includes Operational Transformation, AI and Optimisation problem modelling and solving, with a focus on interactive explainable resource scheduling, planning and simulation. She has a $\mathrm{PhD}$ in $\mathrm{AI}$ and formal computation, and joined $\mathrm{BT}$ in 2000 .

RAPHAEL DORNE works as a Principal Consultant in BT Applied Research. His focus is on applying advanced AI techniques to areas such as Resource Scheduling, Asset Management and Network Design. 